\title{
AstroBot: Um chatbot com inteligência artificial para auxiliar no processo de ensino e aprendizagem de física
}

\author{
Adilmar C. Dantas ${ }^{1}$, Heidie S. Torres ${ }^{2}$, Isabela P. Faria ${ }^{2}$, Wanderson S. Araújo ${ }^{2}$, \\ Guilherme B. dos Santos ${ }^{1}$, Guilherme O. Machado ${ }^{2}$, \\ Adrianne V. de Almeida ${ }^{1}$, Marcelo Z. do Nascimento ${ }^{1}$ \\ ${ }^{1}$ FACOM - Faculdade de Ciência da Computação \\ Universidade Federal de Uberlândia \\ Av. João Naves de Ávila, 2121-38408-100 Uberlândia, MG, Brasil \\ ${ }^{2}$ INFIS - Instituto de Física \\ Universidade Federal de Uberlândia \\ Av. João Naves de Ávila, 2121-38408-100 Uberlândia, MG, Brasil \\ email \{akanehar, heidietorres, isabelafaria.ufu, wandersonsouza392, gui.brilhante, \\ guilhermedeoliveirafacu, verasadrianne3, marcelo.zanchetta\}@gmail.com
}

\begin{abstract}
Resumo. Esse trabalho apresenta um chatbot desenvolvido com tecnologias de processamento de linguagem natural e inteligência artificial para auxiliar no processo de ensino-aprendizagem da disciplina de física. $O$ chatbot foi disponibilizado para os alunos nos atuais aplicativos de mensagens, tais como: WhatsApp, Facebook Messenger e Telegram. A proposta é tornar sua aplicação descontraída e atrativa por meio de aplicações já conhecidas e dominadas por esse público. Esse aplicativo foi avaliado em três turmas do Ensino Médio na tarefa de resolução de exercícios. Com os resultados o docente obteve um feedback das principais dúvidas e questionamentos a respeito do conteúdo didático proposto em sala de aula. Além disso, essa ferramenta permitiu realizar modificações quando necessário, objetivando melhorar o aprendizado dos alunos.
\end{abstract}

\section{Cenário de Uso}

O chatbot pode ser descrito como um programa de computador que tenta simular a troca de mensagens em uma conversação humana. Isso pode ocorrer por meio de texto ou voz de maneira interativa e natural aos usuários [Vassos et al. 2016]. Um dos primeiros chatbots, denominado ELIZA, foi criado em 1966 pelo pesquisador Weizenbaum. A proposta dessa ferramenta era realizar diálogos simples [Weizenbaum 1966, Weizenbaum 1967]. Atualmente, o chatbot pode ser aplicado em uma série de contextos do mundo real.

A utilização das plataformas digitas de comunicação, tais como WhatsApp, WeChat, Telegram e Facebook Messenger se tornaram comum no cotidiano das pessoas. Há uma população das mais diversas faixas etárias que empregam esses aplicativos nas plataformas móveis [Iftene and Vanderdonckt 2016]. De acordo com dados publicados na literatura, investir em recursos e funcionalidades, principalmente educacionais, se tornou interessante uma vez que a familiarização e popularização dessa plataforma já está bem difundida com o publico em geral [Iftene and Vanderdonckt 2016, Dehon et al. 2018].

O uso de Inteligência Artificial (IA) e Processamento de Linguagem Natural (PLN) tem permitido que os chatbots possam ser utilizados em diversas áreas do cotidiano. O conceito de PLN usando IA foi proposto por Unnati Dhavare e Uesh Kulkarni 
VIII Congresso Brasileiro de Informática na Educação (CBIE 2019)

Anais dos Workshops do VIII Congresso Brasileiro de Informática na Educação (WCBIE 2019)

[Dhavare and Kulkarni 2015]. Nessa abordagem, a entrada e saída do sistema podem estar em áudio, textos, imagens ou em uma combinação das mídias. Exemplo de aplicações dessas ferramentas que empregam IA e PLN são os sistemas automatizados de telefonia, suporte educacional e ambientes E-commerces [Abbasi and Kazi 2014, Thomas 2016].

Os chatbots que empregam PLN e IA podem ser explorados na educação para fornecer informações relevantes e personalizadas. Essa solução possibilita ainda: a) realizar busca de conteúdos ou cursos no YouTube, Wikipédia ou Coursera; b) receber notícias de fontes de notícias como feeds de blogs ou contas do Twitter; c) receber suporte de produtividade, como alertas ou lembretes; e d) ser assistente pessoal inteligente para o estudante de maneira interativa e dinâmica [Iftene and Vanderdonckt 2016].

A disciplina de física é considerada abstrata e difícil de ser compreendida pelos estudantes. De acordo com [McDermott 2001], isso acontece devido às metas e realizações dos alunos, o que reflete em uma lacuna entre eles e os professores. Para alguns estudantes, a principal dificuldade está nas metodologias tradicionais empregadas, o que produz muita abstração e ocasiona em uma dificuldade para a compreensão dos fenômenos físicos [Kaufmann and Meyer 2008]. Em [Wieman and Perkins 2005], os autores demonstram a ineficácia da abordagem tradicional no ensino de Física. Os autores mostrando que o ensino tradicional está prejudicando a compreensão da disciplina pelos estudantes pois os alunos normalmente estão memorizando os conceitos, fórmulas e receitas para a solução de problemas. Apesar dos esforços realizados pelos professores para alterar as metodologias e processos de ensino-aprendizagem, os meios encontrados pelos alunos para a aprendizagem de Física acaba se tornando chata, sem motivação e irrelevante.

Baseados nas dificuldades enfrentadas pelos professores para tornar as disciplinas de física atrativa para os alunos, esse trabalho propõem o desenvolvimento de um chatbot com técnicas de inteligência artificial e processamento de linguagem natural para auxiliar no processo de ensino-aprendizagem de Física.

\section{Desenvolvimento}

Antes do desenvolvimento do chatbot, foi realizado um levantamento das principais tecnologias e recursos utilizados recentemente para esse propósito. Para o desenvolvimento da ferramenta, foi definido a utilização da linguagem de programação Python, uma linguagem de programação simples e de fácil aprendizado. Além disso, essa linguagem possui eficientes estruturas de dados de alto nível, e com uma abordagem simples e eficaz, para programação orientada a objetos. A sintaxe elegante e a tipagem dinâmica do Python, juntamente com sua natureza interpretada, fazem dela uma linguagem ideal para o script, permitindo assim o rápido desenvolvimento de aplicativos em diversas áreas e na maioria das plataformas [Van Rossum and Drake Jr 1995]. Para permitir que o chatbot seja disponível para diversos meios de comunicação e sem interrupções, podendo ser acessado a qualquer momento, neste trabalho foi implementado uma arquitetura baseada em nuvem (ver Figura 1).

Para garantir o bom funcionamento do chatbot, a aplicação foi dividida em módulos, tais como: base de dados, IA e PLN. Cada módulo tem suas funcionalidades distribuídas e permite compartilha recursos por meio da computação em nuvem. Para o aperfeiçoamento e treinamento do Chatbot foi utilizado técnicas de IA, por meio 
VIII Congresso Brasileiro de Informática na Educação (CBIE 2019)

Anais dos Workshops do VIII Congresso Brasileiro de Informática na Educação (WCBIE 2019)

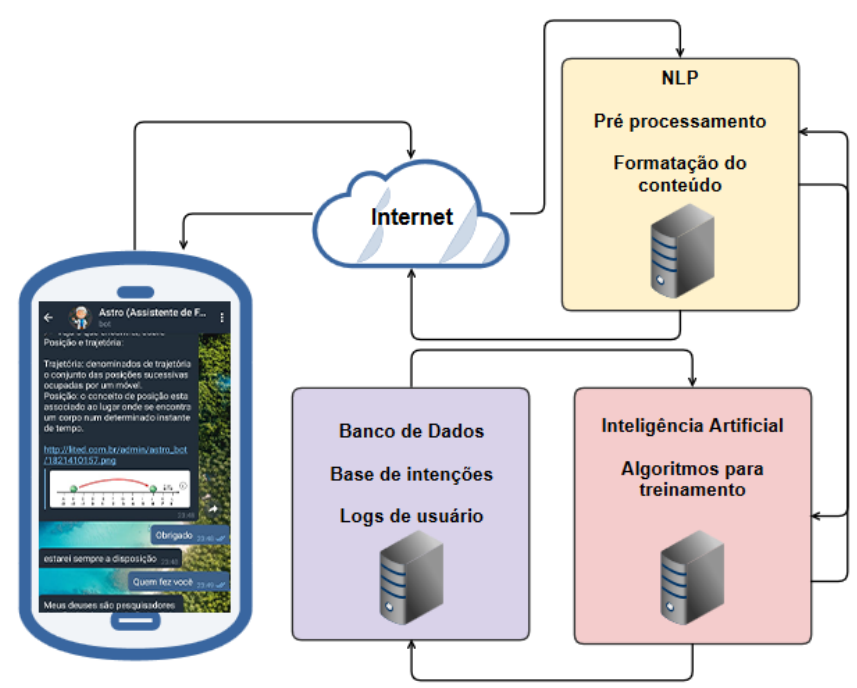

Figura 1. Fluxograma do chatbot e seus respectivos módulos.

de PLN. Essa técnica permite que sistemas sejam capazes de aprender uma linguagem natural dos seres humanos. Esses sistemas de geração de língua natural convertem as informações do banco de dados em linguagem compreensível ao ser humano e assim os sistemas de compreensão de língua natural convertem as ocorrências de linguagem humana em representações mais formais e manipuláveis por programas de computadores [Russell and Norvig 2016].

Para a construção da base de intenções foi utilizado o Dialogflow, uma tecnologia criado pelo Google para trabalhar com PLN por meio de interfaces que permitem a criação de modelos de experiência de conversação com o usuário [Google 2019]. Na Figura 2 é apresentado informações da fase do PLN para elaboração das intenções de dialogo.

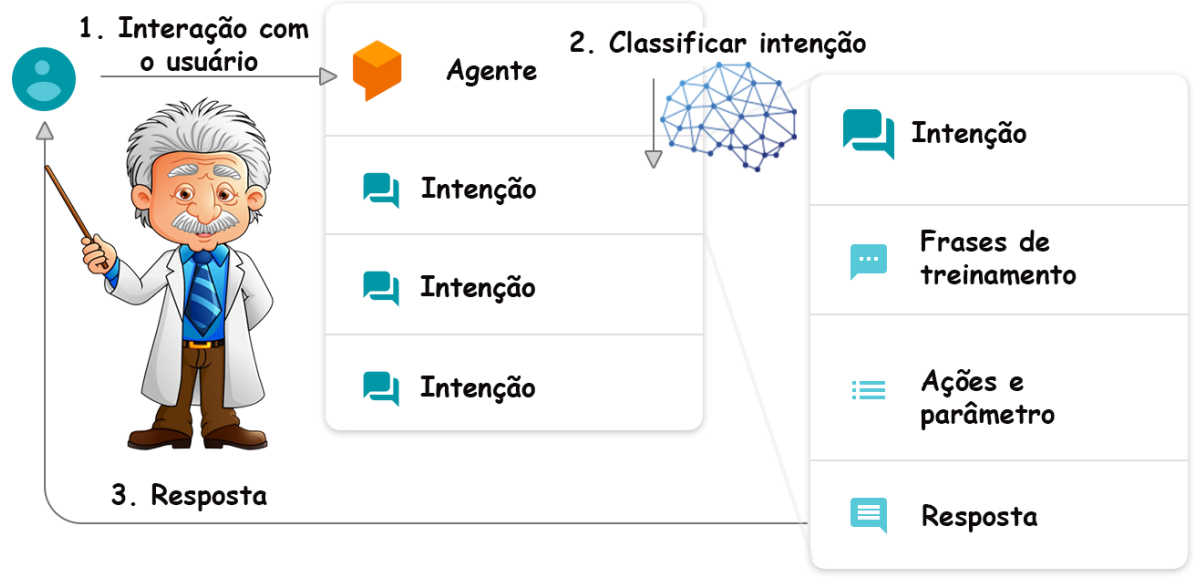

Figura 2. Diagrama de funcionamento do Dialogflow e seus respectivos módulos. Adaptado de: [Google 2019].

O uso dessa tecnologia permitiu que todos os profissionais, os tutores e os docentes, participassem da construção dos diálogos básicos do chatbot. Para a construção do conteúdo didático, referente aos assuntos abordados no ensino médio para a disciplina de física elaborada para execução no chatbot, foi desenvolvido uma aplicação para inserção 
VIII Congresso Brasileiro de Informática na Educação (CBIE 2019)

Anais dos Workshops do VIII Congresso Brasileiro de Informática na Educação (WCBIE 2019)

de conteúdo que permitisse treinar o bot com esses conhecimentos específicos, conforme demonstrado na Figura 3.

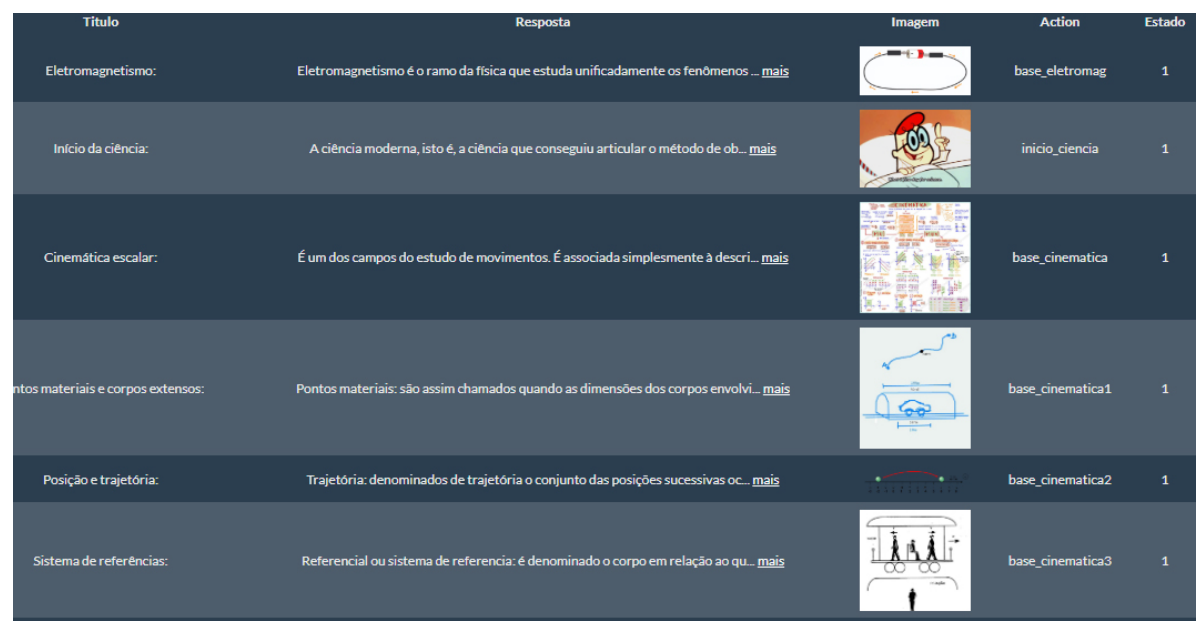

Figura 3. Interface para o gerenciamento de conhecimento didático no sistema chatbot.

Após a inserção do conteúdo e aprendizagem na base do chatbot, ocorreu a etapa de PLN, a qual tinha como objetivo permitir as interações com o usuário. Esse tipo de interface facilita a manutenção e como consequência o aprimoramento da base de conhecimento da aplicação.

\section{Apresentação do software}

O chatbot apresentado neste trabalho pode ser acessado por diferentes aplicativos de comunicação, tais como: WhatsApp, Facebook Messenger e Telegram. Atualmente, essas plataformas podem ser acessadas tanto de dispositivos móveis como também em computadores pessoais, o que permite um aumento do número de usuários que podem ter acesso. Nessa aplicação, a primeira interação que ocorre entre um aluno e o chatbot é uma mensagem de saudação, conforme apresentado na Figura 4(a).

Na Figura 4(b) apresenta um exemplo de interação com usuário por meio da utilização de recursos multimídia como imagens, áudio e vídeos. . Observe que o chatbot deixa o usuário totalmente livre para perguntar sobre qualquer assunto relacionado à disciplina de física e o algoritmos consegue tratar essas questões com PLN. Essa interação só foi possível devido ao desenvolvimento da interface responsável por gerenciar e treinar os conhecimentos a respeito da área em estudo.

Além de ser capaz de interagir com os alunos por meio do PLN, as intenções, questões, não compreendidas pela aplicação são armazenadas em uma base de dados. $\mathrm{O}$ aplicativo permite que essas intenções sejam analisadas pela equipe de docentes e tutores, que avaliam a necessidade da inserção de novos conhecimentos no chatbot. Caso isso aconteça, um novo treinamento deve ser realizado para essas novas questões. Uma outra característica importante nesse aplicativo foi o uso do Dialogflow que permitiu construir um fluxo de dialogo dos alunos em que permite analisar os históricos dos diálogos desde a interação inicial até a resposta conforme exibido na Figura 5. 
VIII Congresso Brasileiro de Informática na Educação (CBIE 2019)

Anais dos Workshops do VIII Congresso Brasileiro de Informática na Educação (WCBIE 2019)

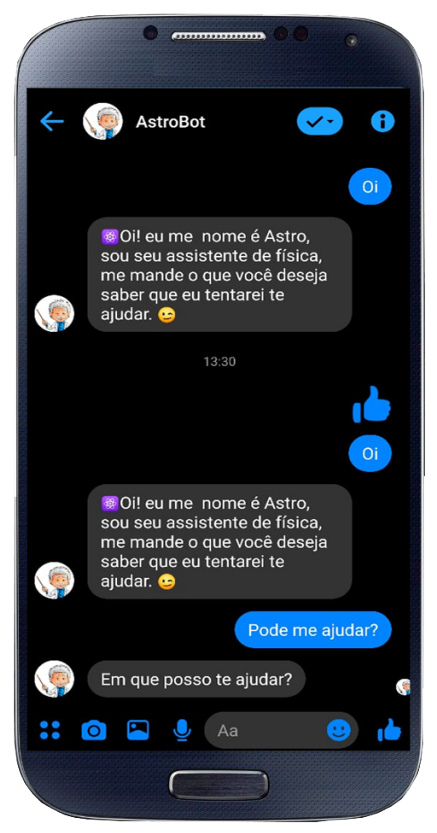

(A)

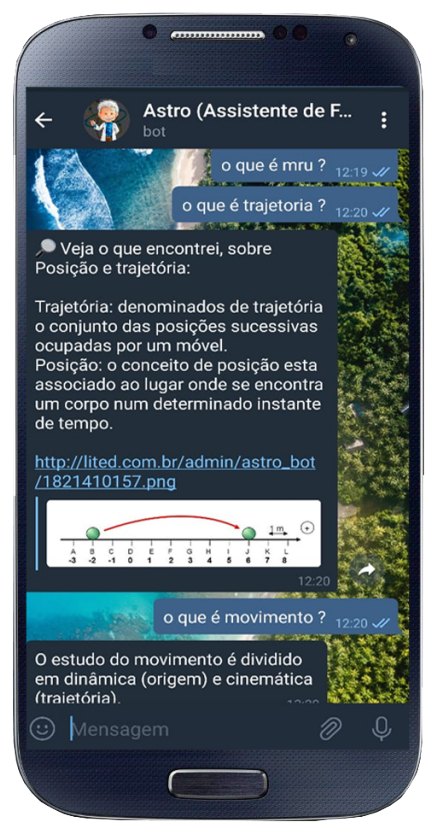

(B)

Figura 4. Exemplos de interações com o chatbot. (a) saudação, (b) apresentação de conteúdo de Física com mídias digitais.

Por meio da interface exibida na Figura 5 é possível obter relatórios detalhados a respeito de: os conteúdos mais questionados pelos alunos, tempo para solucionar as dúvidas, quantidade de questões, etc. Essas informações fornecem ao docente suporte para que possa realizar os ajustes necessários nas aulas de acordo com essas informações.

\section{Avaliação do Chatbot}

\subsection{Investigação do Número de Usuários}

A avaliação do funcionamento e desempenho do chatbot foi realizado por meio de um experimento com o objetivo de avaliar a capacidade de processamento simultâneo da

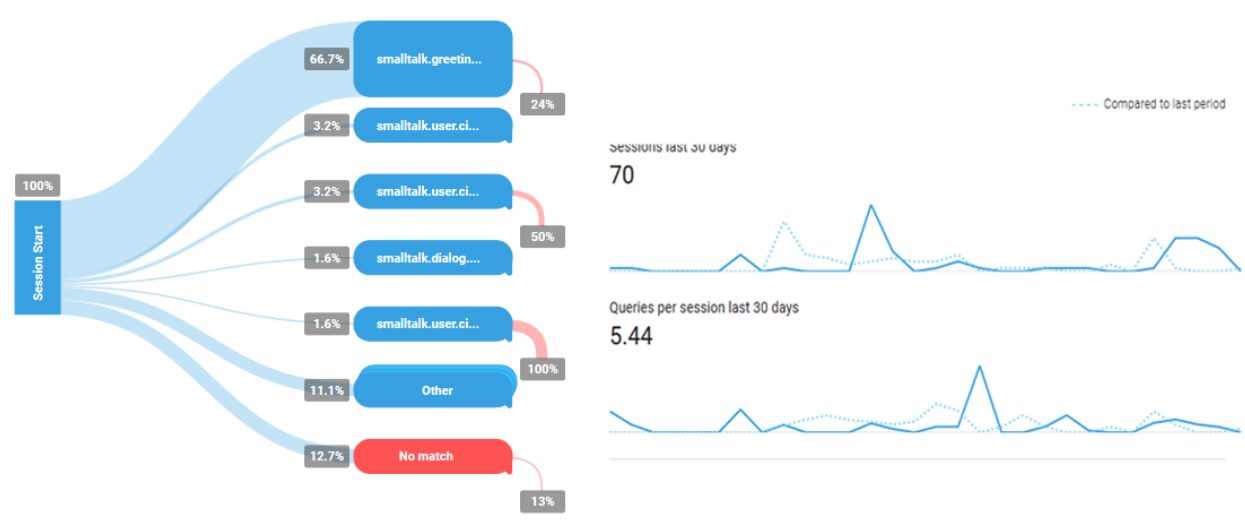

Figura 5. Interface para relatórios dos fluxos dos diálogos processados pela aplicação. 
VIII Congresso Brasileiro de Informática na Educação (CBIE 2019)

Anais dos Workshops do VIII Congresso Brasileiro de Informática na Educação (WCBIE 2019)

aplicação para um cenário de 100, 1000 e 10.000 usuários conectados simultaneamente. Durante a realização desse experimento, o tempo de resposta e a taxa de erro de aplicação foram analisadas com objetivo de verificar o desempenho sobre o número de usuários. $\mathrm{Na}$ Tabela 1 são apresentados os resultados obtidos a partir desse experimento.

Tabela 1. Análise de desempenho do chatbot por requisição.

\begin{tabular}{|c|c|c|}
\hline $\mathbf{N}^{\mathbf{0}}$ Requisições & Tempo de resposta (ms) & Quantidade de erro \\
\hline 100 & 389,00 & 0 \\
\hline 1000 & 443,83 & 0 \\
\hline 10000 & 1066,33 & 3 \\
\hline 100000 & 3688,69 & 18 \\
\hline Média & 1396,96 & 5,25 \\
\hline
\end{tabular}

Esse experimento mostra que mesmo com muitas requisições simultâneas, a aplicação foi capaz de processar corretamente as intenções e fornecer a resposta aos usuários em um tempo médio de 1396,96 milissegundos e com taxa de erro de 5,25. Isso mostra que aplicação é interessante para ajudar estudantes no processo de aprendizagem de física com tempo de resposta considerado satisfisfatório.

\subsection{Investigação da Ferramenta para Ensino}

Para que fosse possível realizar a avaliação da aplicação o mesmo foi submetido ao Comitê de Ética em Pesquisa de acordo com as conformidades da Universidade Federal de Uberlândia. Para critério de avaliação, o chatbot foi aplicado em quatro turmas do primeiro ano do ensino médio, na disciplina de física, como um total de 25 alunos. Nesse experimento, os alunos foram, primeiramente, orientados a como utilizar a aplicação para a disciplina durante a realização de execícios em fora de sala de aula durante quatro semanas. Essa etapa tinha como objetivo auxiliar os alunos na realização de suas atividades por meio do uso dessa tecnologia, e realizar o levantamento das principais dúvidas e questionamentos.

Durante as quatro semanas o chatbot recebeu um total de 72 questionamentos dos estudantes como é exibido no gráfico na Figura 6. Nesta figura é possível observar as principais intenções processadas durante a sua disponibilização.

Nos resultados da Figura 6, é possível ver que as intenções mais requisitadas foram as relacionadas: vetores $(25,0 \%)$, movimento uniformemente variado $(18,75 \%)$, trajetória $(15,63 \%)$, pode me ajudar $(14,6 \%)$ e variáveis $(9,38 \%)$. Esse levantamento foi aproveitado pelo docente para auxilia-lo no processo de aprimoramento do plano de aula, dando prioridade às dúvidas e questionamentos obtidos por meio do chatbot.

A segunda etapa do experimento foi responsável por avaliar a viabilidade da tecnologia e quais as expectativas dos alunos em relação ao uso dessa ferramenta. Para essa avaliação foi aplicado um questionário com oito perguntas a respeito do chatbot. Na Tabela 2 são apresentados as alternativas: concordo totalmente, concordo parcialmente, neutro, discordo parcialmente e discordo totalmente. As respostas obtidas para as questões são apresentados na Tabela 3. 
VIII Congresso Brasileiro de Informática na Educação (CBIE 2019)

Anais dos Workshops do VIII Congresso Brasileiro de Informática na Educação (WCBIE 2019)

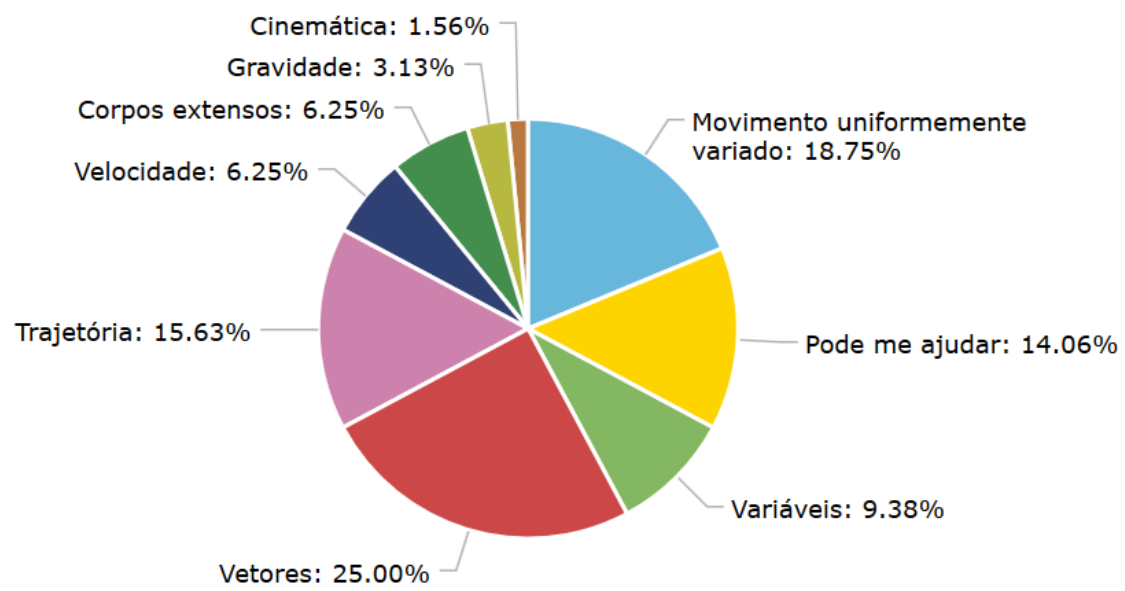

Figura 6. Resultados das principais intenções processadas pelo chatbot durante o uso pelos estudantes.

Tabela 2. Questões elaboradas para o questionário avaliativo do chatbot.

\begin{tabular}{ll}
\hline Questão & \\
\hline Eu gostei de utilizar o Astro chatbot & $\begin{array}{l}\text { Usar o chatbot pode aumentar meu desempenho } \\
\text { quanto ao planejamento das minhas atividades acadêmicas }\end{array}$ \\
É fácil utilizar o chatbot & $\begin{array}{l}\text { O chatbot produz os resultados que espero de uma ferramenta } \\
\text { de suporte à comunicação entre professores e alunos }\end{array}$ \\
O visual do chatbot é agradável & $\begin{array}{l}\text { Eu pretendo continuar utilizando o chatbot na disciplina de Física } \\
\text { O chatbot pode aumentar meu desempenho } \\
\text { Irei recomendar o chatbot para meus amigos } \\
\text { no processo de ensino e aprendizado }\end{array}$ \\
\hline
\end{tabular}

\section{Considerações finais}

Esse trabalho apresentou o desenvolvimento e aplicação de um chatbot com uso de IA para auxiliar no processo de ensino-aprendizagem da disciplina de física. Através da realização de experimentos foi possível demonstrar que, conforme apresentado pela literatura, a ferramenta de chatbot é uma tecnologia que permite colaborar com o processo de ensino e aprendizagem. Os resultados confirmaram as hipóteses levantadas sobre as expectativas dos alunos em relação à utilização dessa tecnologia.

Além disso, os dados produzidos a respeito das intenções mais acessadas, ou seja, as principais dúvidas e questionamentos levantadas pelos alunos no momento da resolução de exercícios, contribuiu efetivamente para o desenvolvimento das aulas produzidas pelos docentes. Em trabalhos futuros, pretende-se avaliar o rendimento e evolução

Tabela 3. Resultados das respostas dos questionários aplicados aos 25 alunos.

\begin{tabular}{cccccc}
\hline Questão & Concordo Totalmente & Concordo Parcialmente & Neutro & Discordo Parcialmente & Discordo Totalmente \\
\hline 1 & $44,00 \%$ & $28,00 \%$ & $28,00 \%$ & - & - \\
2 & - & $68,00 \%$ & $20,00 \%$ & - & $12,00 \%$ \\
3 & $36,00 \%$ & $44,00 \%$ & $20,00 \%$ & - & - \\
4 & $60,00 \%$ & $40,00 \%$ & - & - & - \\
5 & $28,00 \%$ & $48,00 \%$ & $24,00 \%$ & - & - \\
6 & $40,00 \%$ & $36,00 \%$ & $24,00 \%$ & - & - \\
7 & $44,00 \%$ & $28,00 \%$ & $28,00 \%$ & - & - \\
8 & $44,00 \%$ & $28,00 \%$ & $28,00 \%$ & - & - \\
\hline
\end{tabular}


VIII Congresso Brasileiro de Informática na Educação (CBIE 2019)

Anais dos Workshops do VIII Congresso Brasileiro de Informática na Educação (WCBIE 2019)

dos alunos com a utilização do chatbot. Permitindo obter novas contribuições e possíveis limitações quanto à aplicação.

\section{Referências}

Abbasi, S. and Kazi, H. (2014). Measuring effectiveness of learning chatbot systems on student's learning outcome and memory retention. Asian Journal of Applied Science and Engineering, 3(2):251-260.

Dehon, P., Silva, A., Inocêncio, A. C., Castro, C., Costa, H., and Júnior, P. P. (2018). Cvchatbot: Um chatbot para o aplicativo facebook messenger integrado ao ava moodle. In Brazilian Symposium on Computers in Education (Simpósio Brasileiro de Informática na Educação-SBIE), volume 29, page 1623.

Dhavare, U. and Kulkarni, U. (2015). Natural language processing using artificial intelligence. International Journal of Emerging Trends \& Technology in Computer Science (IJETTCS), 4(2):203-205.

Google (2019). Overview dialogflow @ONLINE.

Iftene, A. and Vanderdonckt, J. (2016). Moocbuddy: a chatbot for personalized learning with moocs. In RoCHI-International Conference on Human-Computer Interaction, page 91 .

Kaufmann, H. and Meyer, B. (2008). Simulating educational physical experiments in augmented reality. ACM.

McDermott, L. C. (2001). Oersted medal lecture 2001:"physics education research-the key to student learning". American Journal of Physics, 69(11):1127-1137.

Russell, S. J. and Norvig, P. (2016). Artificial intelligence: a modern approach. Malaysia; Pearson Education Limited,.

Thomas, N. (2016). An e-business chatbot using aiml and lsa. In 2016 International Conference on Advances in Computing, Communications and Informatics (ICACCI), pages 2740-2742. IEEE.

Van Rossum, G. and Drake Jr, F. L. (1995). Python tutorial. Centrum voor Wiskunde en Informatica Amsterdam, Netherlands.

Vassos, S., Malliaraki, E., dal Falco, F., Di Maggio, J., Massimetti, M., Nocentini, M. G., and Testa, A. (2016). Art-bots: Toward chat-based conversational experiences in museums. In International Conference on Interactive Digital Storytelling, pages 433-437. Springer.

Weizenbaum, J. (1966). Eliza - a computer program for the study of natural language communication between man and machine. Communications of the ACM, 9(1):36-45.

Weizenbaum, J. (1967). Contextual understanding by computers. Communications of the ACM, 10(8):474-480.

Wieman, C. and Perkins, K. (2005). Transforming physics education. Physics today, 58(11):36. 